\title{
Determination of line edge roughness in low-dose top-down scanning electron microscopy images
}

\author{
Thomas Verduin, ${ }^{*}$ Pieter Kruit, and Cornelis W. Hagen \\ Delft University of Technology, Faculty of Applied Sciences, Department of Imaging Physics, Charged Particle Optics Group, \\ Lorentzweg 1, 2628 CJ Delft, The Netherlands
}

\begin{abstract}
We investigated the off-line metrology for line edge roughness (LER) determination by using the discrete power spectral density (PSD). The study specifically addresses low-dose scanning electron microscopy (SEM) images in order to reduce the acquisition time and the risk of resist shrinkage. The first attempts are based on optimized elliptic filtering of noisy experimental SEM images, where we use threshold-based peak detection to determine the edge displacements. The effect of transversal and longitudinal filterings cannot be ignored, even when considering an optimized filter strength. We subsequently developed a method to detect the edge displacements without the use of a filter and thus avoiding biasing. This makes it possible to study how much image noise is acceptable and still determine the LER. The idea is to generate random images of line edges using the model of Palasantzas and the algorithm of Thorsos. We study the simulated PSDs as a function of the number of line edges and report on the convergence of the parameters (LER, correlation length, and roughness exponent) by fitting the Palasantzas model extended with a white noise term. This study demonstrates that a very noisy image with 12 line edges and about 2 electrons per pixel on average (charge density $\approx 10 \mu \mathrm{C}$ ) already produces an estimation for LER with a relative error (one-sigma) of about $10 \%$. Furthermore, increasing the dose beyond 20 electrons per pixel does not significantly improve the LER determination. ๑ 2014 Society of Photo-Optical Instrumentation Engineers (SPIE) [DOI: 10.1117/1.JMM. 13.3.033009]
\end{abstract}

Keywords: critical dimension-scanning electron microscopy metrology; line edge roughness; power spectral density.

Paper 14066P received May 1, 2014; revised manuscript received Jun. 28, 2014; accepted for publication Jul. 17, 2014; published online Aug. 13, 2014.

\section{Introduction}

The determination of line edge roughness (LER) becomes increasingly important as semiconductor devices decrease in dimensions. ${ }^{1-3}$ This results in smaller tolerances on LER determination and as a consequence, the metrology becomes more critical. There are two classes of LER metrology. One is on-line metrology, which is typically performed in scanning electron microscopy (SEM) combined with dedicated (proprietary) software for LER analysis. Another class is off-line metrology and only deals with the image analysis. The latter is used, for instance, for resist characterization. Typically, in off-line LER analysis, the fluctuations in edge displacements are determined using a thresholdbased peak detector, a Canny-edge detection filter, or by a homemade edge detection algorithm (see, for example, Ref. 4). Although the edge displacements already give a direct measurement of the roughness, the LER is best analyzed by the power spectral density (PSD). ${ }^{5-9}$ There are a couple of problems related to this type of determination. First of all, there are statistical and systematic errors because the actual PSD is approximated by sampling the edge displacements of a pattern with a finite number of measurement intervals. The statistical errors are, for example, described in Refs. 7 and 8 . The systematic errors have recently been studied in Ref. 9. There is also the problem of shrinkage, where the act of measuring the edge displacements by irradiation with an e-beam induces changes in the pattern (see, for example, Refs. 8 and 10-12). Finally, there is the problem of biasing due to the use of a filter prior to the edge detection

*Address all correspondence to: Thomas Verduin, E-mail: T.Verduin @ tudelft.nl in noisy SEM images (see, for example, Refs. 4, 7, 10, and 13-15). In this article, we focus on off-line metrology and investigate how much image noise is acceptable by decreasing the dose in simulated top-down SEM-like images. The determination of LER is a delicate issue, and we ask the following questions: Can we reduce the influence of a filter to negligible levels by carefully optimizing the filter strength? Is it possible to avoid filtering in low-dose images at all? How many low-dose images of the line edges are required for estimating LER?

\section{Line Edge Determination}

Let us begin with a typical example of a top-down SEM image of line edges, which is given in Fig. 1. This image was recorded by J. Jussot from CNRS-LTM/CEA-LETI in 2012 using a critical dimension(CD)-SEM from Hitachi. The properties of the resist are unknown to us due to the disclosure restrictions. These resist properties, however, are of no further interest to us.

In Fig. 2(a), we show the amplitude of a single horizontal scan-line taken from the center of Fig. 1. The noise in Fig. 2(a) is due to the pixel noise and corresponds to 16 integration frames. The number of integration frames should be kept as low as possible to reduce the effect of resist shrinkage. ${ }^{8,10-12}$ However, detection algorithms, such as thresholdbased detectors or Canny-edge detection filters, often do not find the edge or find too many edges in such noisy data. Working with low-noise images has two problems: they take a long time to accumulate and there is a risk of 


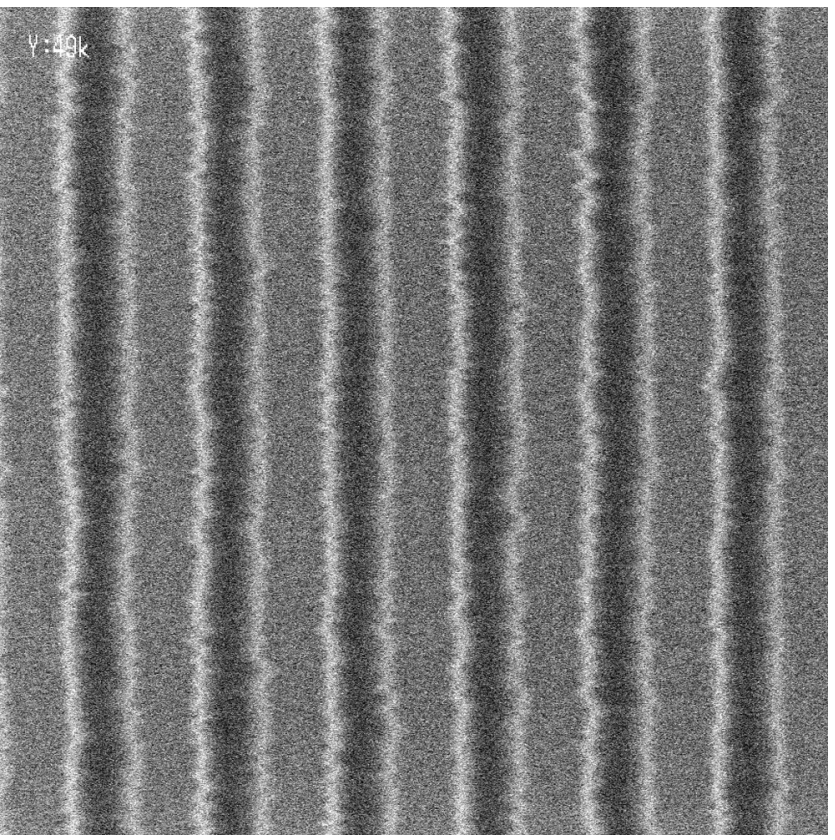

Fig. 1 A typical top-down SEM image of line edges, which was recorded by J. Jussot from CNRS-LTM/CEA-LETI in 2012 using a CD-SEM from Hitachi. This image is recorded with a probe current of $6.0 \mathrm{pA}$ at an acceleration voltage of $500 \mathrm{~V}$. The number of integration frames equals 16 . The magnification is such that the field size is $2.76 \mu \mathrm{m}$ in length (1024 px) and 450-nm wide (1024 px). The resulting pixel size is approximately $2.7 \mathrm{~nm} \times 0.44 \mathrm{~nm}$.

resist shrinkage. An obvious way to reduce the noise is to apply a filter to the recorded image. The result of applying a symmetric Gaussian filter on the amplitude is shown in Fig. 2(b). The edge displacements can be determined by a threshold detector if the noise in the amplitude is sufficiently reduced.

The discrete PSD of the edge displacements is obtained by calculating the complex amplitude of the Fourier coefficients

$P_{n}=\frac{L}{2 \pi}\left|F_{n}\right|^{2}$,

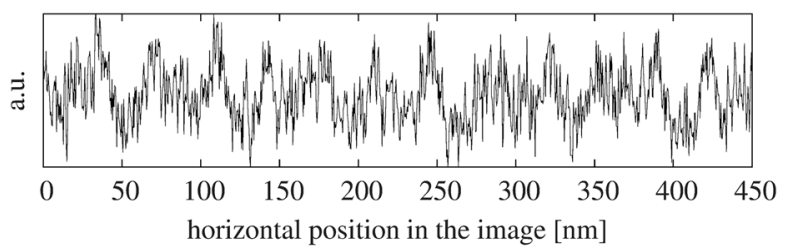

(a)

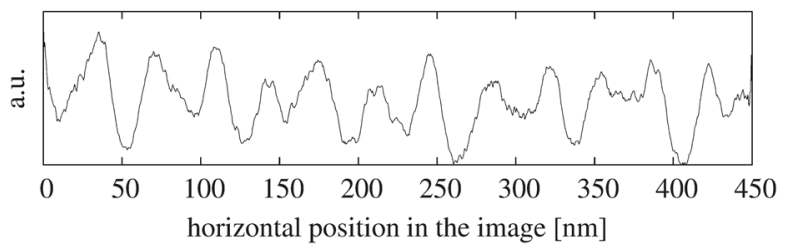

(b)

Fig. 2 The amplitude of a single horizontal scan-line taken from the center of Fig. 1. The signal in (a) represents the amplitude directly from the image without any postprocessing. This raw signal is too noisy for threshold-based edge detection. However, the noise of this signal can be reduced by applying a symmetric Gaussian filter to the image and the result on the amplitude is demonstrated in (b). where the discrete Fourier transform is determined as

$F_{n}=\frac{1}{N} \sum_{j=0}^{N-1}\left(x_{j}-\langle x\rangle_{N}\right) \exp \left(-i k_{n} j \Delta y\right)$,

where $N$ is the number of sampled edge displacements, $x_{j}$ is the displacement of the $j$ th edge position, $\langle x\rangle_{N}$ is the mean position of the edge, $\Delta y$ is the measurement interval, and $k_{n}$ is the discrete wave number

$k_{n}=\frac{2 \pi n}{L}$,

where $n=0,1,2, \ldots, N-1$, and $L$ is the length of the edge. The variance of the edge displacements is related to the PSD by

$\sigma^{2}=\frac{2 \pi}{L} \sum_{n=0}^{N-1} P_{n}$

which is Parseval's relation. The discrete PSD given by Eq. (1) is only an approximation to the actual spectrum of a quasiinfinite long line and the finite line length $L$ is a source of statistical noise (see, for example, Refs. 7 and 8). The statistical noise in the discrete PSD can be reduced by averaging over many line edges

$P_{n}^{*}=\frac{L}{2 \pi}\left\langle\left|F_{n}\right|^{2}\right\rangle_{N^{*}}$

where $N^{*}$ counts the number of line edges over which the PSD is averaged. This averaging is possible if the length of the line edge is much longer than the correlation length of the edge fluctuations.

We calculate the discrete PSD of the line edges shown in Fig. 1 and, in order to reduce the noise in the power spectrum, average the PSDs of the 50 recorded SEM images of line edges of the same kind. Every individual SEM image is at first convoluted with an elliptic Gaussian filter

$\widetilde{I}(x, y)=I(x, y) * G(x, y)=I(x, y) * \frac{\exp \left(-\frac{x^{2}}{2 \sigma_{\mathrm{T}}^{2}}-\frac{y^{2}}{2 \sigma_{\mathrm{L}}^{2}}\right)}{2 \pi \sigma_{\mathrm{T}} \sigma_{\mathrm{L}}}$,

where $\widetilde{I}$ is the filtered SEM image, $I$ is the original SEM image, and $G$ is the elliptic filter. The strength of the elliptic filter in the transverse direction to the edges is $\sigma_{\mathrm{T}}$ and the strength in the longitudinal direction is $\sigma_{\mathrm{L}}$. The edge displacements are then determined using a threshold-based edge detection algorithm. In this method, we identify an edge whenever the normalized amplitude of a peak is larger than 0.3 with respect to the noise level. This threshold is obtained empirically by analyzing many images. The choice of a particular threshold level is subjective and we will discuss the effect of this on our results later. The directional strengths of the filter are optimized on a per image basis and are such that every scan-line of a SEM image produces the real number of edges in the image. In other words, if we decrease the strength of the elliptic filter even further, then we will count more or fewer edges than are physically present in the pattern. This happens because there are peaks within the signal [see Fig. 2(a)] which are not related to the edges but are related to the image noise. As we decrease the 
strength of the elliptic filter, the amplitudes of the peaks from the image noise increase and at some point, we can no longer separate them from the peak due to a physical edge. It is at this point that the threshold method of determination becomes indecisive: it can no longer decide which peak is an edge and which peak is not.

Suppose, for a particular SEM image of line edges, that we check a range of longitudinal and transversal filter strengths whether or not the edge detection method produces the correct number of edges for all scan-lines. There exists a subdomain where the edge detection method produces the correct number of edges and a complementary domain where it does not produce the correct number of edges. This idea is illustrated in Fig. 3, in which the marked region corresponds to the domain where the number of detected edges does not match (at least for one scan-line) the real number of edges. We define a filter to be optimized whenever it is on this boundary.

We note that an optimized filter is preferred because a filter not only reduces the image noise, but also destroys the frequency content related to the actual edge displacements. The idea of an optimized filter is to minimize this effect. A point on the boundary of Fig. 3 can be found, for example, by bisective optimization. The idea is to start with a predefined minimal and maximal filter strengths and to split the interval in half as long as the number of edges is correct. This procedure is repeated until the resulting interval convergences sufficiently to a point on the boundary. In this way, we have found for the 50 recorded SEM images, the following optimized directional filter strengths: $\sigma_{\mathrm{T}}=2.5 \mathrm{px} \pm 0.87 \mathrm{px}$ and $\sigma_{\mathrm{L}}=3.6 \mathrm{px} \pm 0.69 \mathrm{px}$. We emphasize that this particular filter strength relates to just one arbitrary point on the boundary of Fig. 3. However, we attempt to demonstrate that the PSD is affected, even though we optimize our filter strength. In this respect, any point on the boundary is acceptable as long as we are consistent in our method of

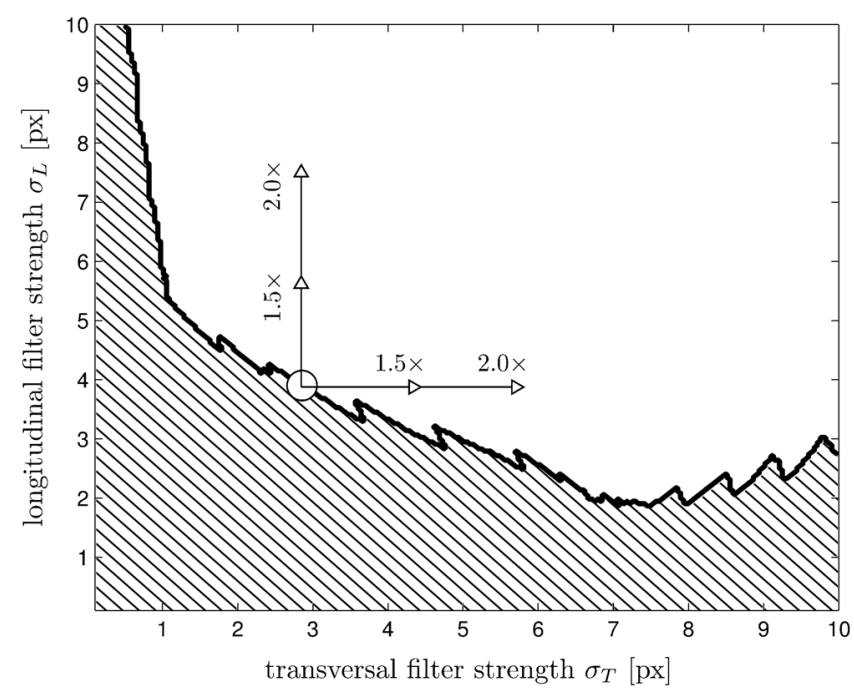

Fig. 3 The effect of filtering on the number of detected edges in Fig. 1. The longitudinal filter strength $\sigma_{\mathrm{L}}$ is shown on the vertical axis, and the transversal filter strength $\sigma_{T}$ is shown on the horizontal axis. The marked region corresponds to a domain where the number of detected edges does not match the real number of edges (12). The open marker on the boundary represents one of the possible optimized filter settings, and the arrows indicate how the directional filter strength is adjusted. determination. In Fig. 4(a), we increase the strength of the filter in the transverse direction, while the longitudinal strength remains fixed at the optimized value. Similarly, in Fig. 5(a), we only increase the strength of the filter in the longitudinal direction. The arrows in Fig. 3 show how the directional filter strength is adjusted. In Figs. 4(b) and 5(b), we plot the square root of the cumulative sum of the PSD from the lowest wave number toward the highest wave number. This cumulative sum gives the variance via Parseval's equation [Eq. (4)] and by taking the square root, we can see how the standard deviation develops as a function of increasing wave numbers.

In Figs. 4(a) and 5(b), we observe an undesired effect of the filtering on the PSD: besides suppression of the high frequencies (which is required to reduce the image noise), the center frequencies (near $k=10^{-1}$ ) are affected as well. This conclusion (filtering biases the result) is drawn by various authors as well (see, for example, Refs. 4, 7, 10, and 13-15). Here, we show that this effect, for both transversal and longitudinal filterings, cannot be ignored, even when considering an optimized filter strength. This is best seen in the cumulative sums in Figs. 4(b) and 5(b), where the total roughness is shown for different filter strengths. We have summarized the effect on LER in Table 1 for varying directional filter strengths. Several

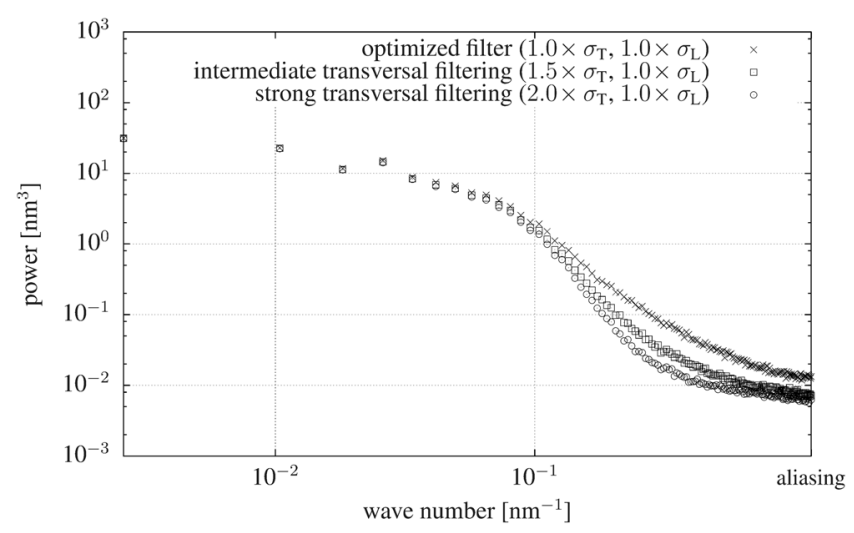

(a)

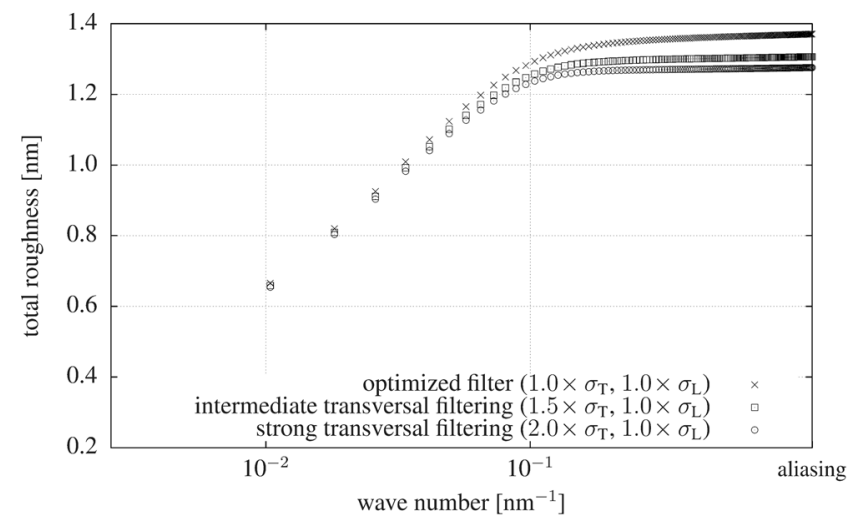

(b)

Fig. 4 The effect of transverse filtering on the discrete PSD and the total roughness is shown. The results are obtained from analyzing the set of 50 experimental SEM images recorded by J. Jussot. The PSD is given in (a) and the total roughness is given in (b), which is obtained by taking the square root of the cumulative sum of the PSD. The markers correspond to different transversal strengths with respect to the optimized elliptic filter. 


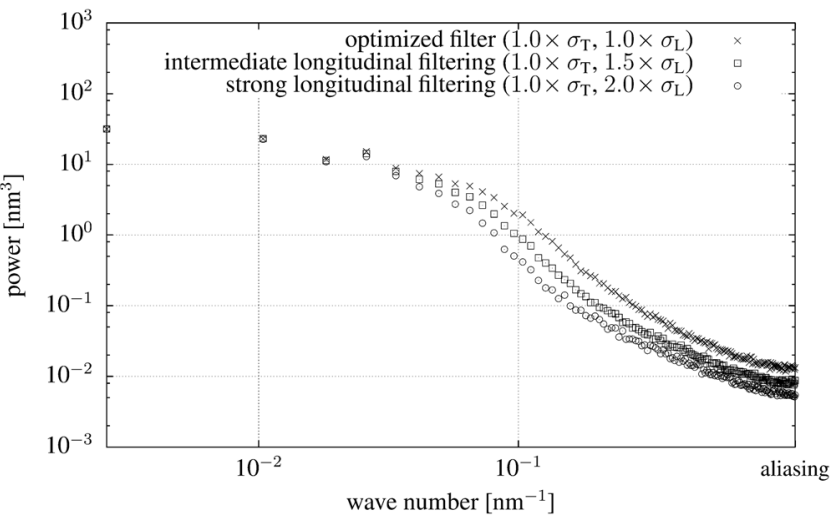

(a)

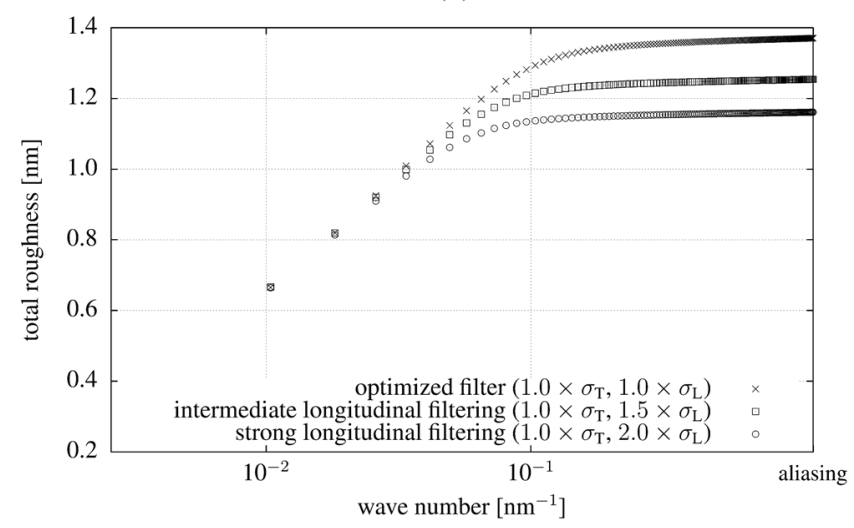

(b)

Fig. 5 The effect of longitudinal filtering on the discrete PSD and the total roughness is shown. The results are obtained from analyzing the set of 50 experimental SEM images recorded by J. Jussot. The PSD is given in (a) and the total roughness is given in (b), which is obtained by taking the square root of the cumulative sum of the PSD. The markers correspond to different longitudinal strengths with respect to the optimized elliptic filter.

attempts have been made to correct this biasing (see, for example, Refs. 8, 10, 11, and 15-17). The problem is, however, that these attempts do not capture the effect of filtering, instead they capture the effect of image pixel noise.

We now ask the following question: How much further do we need to reduce the strength of the filter (beyond the optimized filter), such that the filter dependency on the measured LER becomes insignificant? We failed in reducing the strength of the filter, because then the edges cannot be

Table 1 LER when using an intermediate or strong filter in, respectively, the transverse or longitudinal direction. The optimized elliptic filter, obtained by analyzing 50 recorded SEM images of line edges, corresponds to a transversal strength of $\sigma_{\mathrm{T}}=2.5 \mathrm{px} \pm 0.87 \mathrm{px}$ and a longitudinal strength of $\sigma_{\mathrm{L}}=3.6 \mathrm{px} \pm 0.69 \mathrm{px}$. The intermediate filter has 1.5 times the strength of the optimized filter in either the transversal or longitudinal direction. Similarly, the strong filter has 2.0 times the strength of the optimized filter.

\begin{tabular}{lcc} 
Filter strength & Transversal $(\mathrm{nm})$ & Longitudinal $(\mathrm{nm})$ \\
\hline Optimized filter & 1.37 & 1.37 \\
Intermediate filter & 1.30 & 1.25 \\
Strong filter & 1.28 & 1.16 \\
\hline \hline
\end{tabular}

detected anymore by our threshold-based peak detection. The interested reader might argue that we could use the threshold level to reduce the strength of the optimized filter even further. It is true indeed that the strength of the optimized filter depends on the particular choice of the threshold level. The threshold level balances the amplitude of the image noise and the amplitude of a peak due to an edge. This must be seen in the context of filtering, where we would like to reduce the filter strength as much as possible. This means that the threshold level should be as low as possible. In addition, the threshold level could be optimized dynamically per line edge, which would probably produce a weaker filter than the optimized filter we have used. In other words, maybe we could reduce the influence of the filter by clever optimization tricks, but how do we know if and when the influence has become insignificant? We conclude that the best solution would be to avoid a filter at all.

We recently developed a method to detect edge displacements without the use of a filter. In fact, this method works without using any postprocessing of the SEM image. Let us explain this method in detail. We approximate the signal profile of the SEM by integrating a recorded SEM image in the direction of the line edges. For example, the approximate SEM signal profile of Fig. 1 is given in Fig. 6.

We emphasize that this is only an approximation, because by integrating in the direction of the line edges, the actual shape of this profile becomes a function of the roughness, which is present in the edges. In principle, the roughness of the edges must be corrected by counter displacing the rows. For the moment, we assume that this roughness dependency can be neglected and that the profile function can be seen as the SEM signal response to a straight edge. We will come back to this issue later. The SEM signal profile of an isolated edge is shown in Fig. 7, which is just one of the 12 peaks shown in Fig. 6.

We model the SEM signal profile of an isolated edge by matching two vertically shifted and normalized Gaussians at the center, which has the following mathematical representation:

$P(x)=\left\{\begin{array}{ll}b_{\mathrm{L}}+\left(1-b_{\mathrm{L}}\right) \exp \left(-\frac{1}{2} \frac{(x-\mu)^{2}}{\sigma_{\mathrm{L}}^{2}}\right) & x<\mu \\ b_{\mathrm{R}}+\left(1-b_{\mathrm{R}}\right) \exp \left(-\frac{1}{2} \frac{(x-\mu)^{2}}{\sigma_{\mathrm{R}}^{2}}\right) & x \geq \mu\end{array}\right.$,

where $b_{\mathrm{L}}$ and $b_{\mathrm{R}}$ define the base level found at the far left and right, respectively, in Fig. 7, and $\sigma_{\mathrm{L}}$ and $\sigma_{\mathrm{R}}$ define the standard deviation to the left and right, respectively, of the center $\mu$. The left base level is defined such that $b_{\mathrm{L}}=\lim _{x \rightarrow-\infty} P(x)$ and the right base is defined such that $b_{\mathrm{R}}=\lim _{x \rightarrow+\infty} P(x)$. Note that if $b_{\mathrm{L}}=b_{\mathrm{R}}=0$ (the Gaussians are not shifted upward) and $\sigma_{\mathrm{L}}=\sigma_{\mathrm{R}}$ (same standard deviation), then

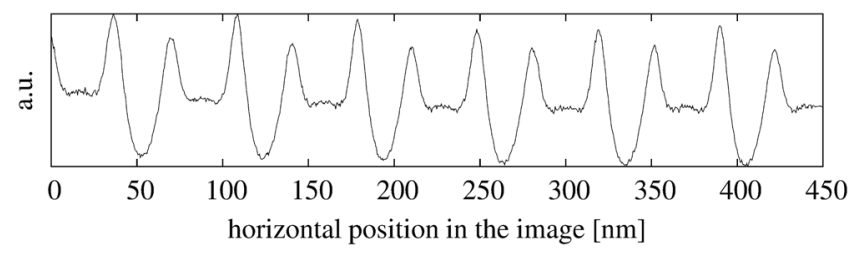

Fig. 6 The SEM signal profile for 12 line edges as obtained by integrating the SEM image of Fig. 1 in the direction of the line edges. 


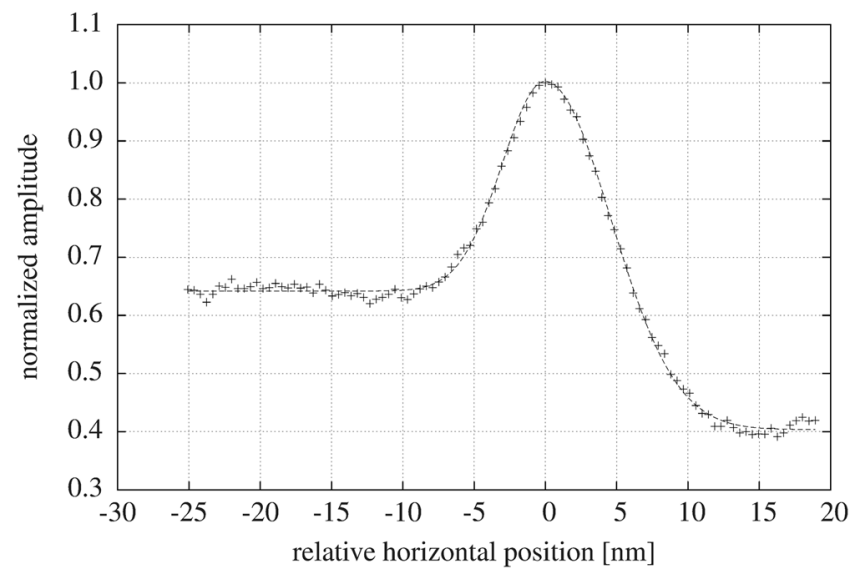

Fig. 7 The SEM signal profile of an isolated edge taken from Fig. 6. The dashed line is the best fit of the model given by Eq. (7).

Eq. (7) reduces to the standard definition of a Gaussian distribution, up to a normalization factor. The parameters $b_{\mathrm{L}}$, $b_{\mathrm{R}}, \sigma_{\mathrm{L}}$, and $\sigma_{\mathrm{R}}$ are fitted against the integrated SEM signal profile. The dashed line in Fig. 7 is the best fit of this model against a single-isolated experimental profile obtained by integrating Fig. 1 in the direction of the line edges. The method of fitting a Gaussian to an integrated SEM image is also found in Ref. 18, where it is used to estimate line widths. This study, however, considers a more complicated fit for the determination of LER.

The idea is now to match this double Gaussian model to every sampled row of a single edge using parameter optimization. We introduce the following degrees of freedom to the model for the SEM signal profile:

$s \cdot P(x-\Delta x)$,

where $s$ scales the profile amplitude $P$, and $\Delta x$ is the transversal displacement of the profile. The parameters $s$ and $\Delta x$ are determined by using an interior trust-region-reflective minimization algorithm. The interested reader is referred to the article of Coleman and $\mathrm{Li}^{19}$ for details on the minimization procedure. In Fig. 8, we demonstrate one of the matching results using the raw unfiltered signal, which is taken directly from the SEM image. Clearly, for such noisy data, a threshold-based edge detection method could not have found the position of the edge.

Now that we can detect the edge displacements without using a filter, we reconsider the set of recorded SEM images from J. Jussot. However, this time, we use our SEM profilebased edge detection. The PSD without using a filter (crossmarkers) is given in Fig. 9(a) together with the optimized filter (square markers) found in Figs. 4(a) and 5(a).

In Fig. 9(a), we observe that the pixel noise really starts to contribute after the marker "noise limit." In Fig. 9(b), we observe that the cumulative sum after the marker accounts for approximately $1.6 \mathrm{~nm}-1.4 \mathrm{~nm}=0.2 \mathrm{~nm}$. Therefore, a crude estimate for the actual LER is $1.4 \mathrm{~nm}$. This is to be compared with the estimate of $1.3 \mathrm{~nm}$ for the optimized filter (square markers) found in Fig. 9(b). Apparently, the optimized filter that we have applied in our first attempt is not that far off. What about the fact that we neglected the roughness in the edges when determining the SEM signal profile? At the moment, the roughness of the edges is effectively absorbed into the parameters (specifically $\sigma_{\mathrm{L}}$ and $\sigma_{\mathrm{R}}$ )

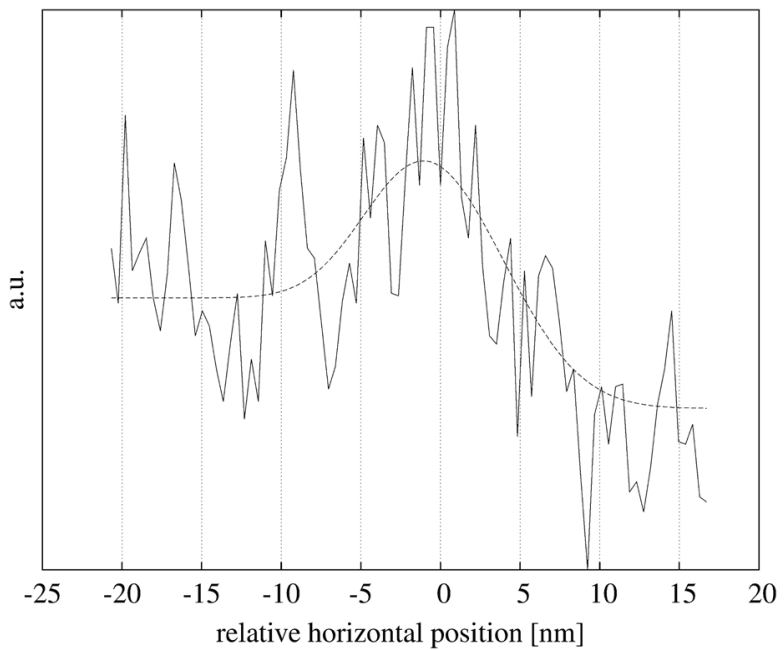

Fig. 8 The matching of the model for the SEM signal profile in the raw (unfiltered) amplitude of an edge in a SEM image is illustrated. The profile is matched by varying the transversal displacement and amplitude of the profile function.

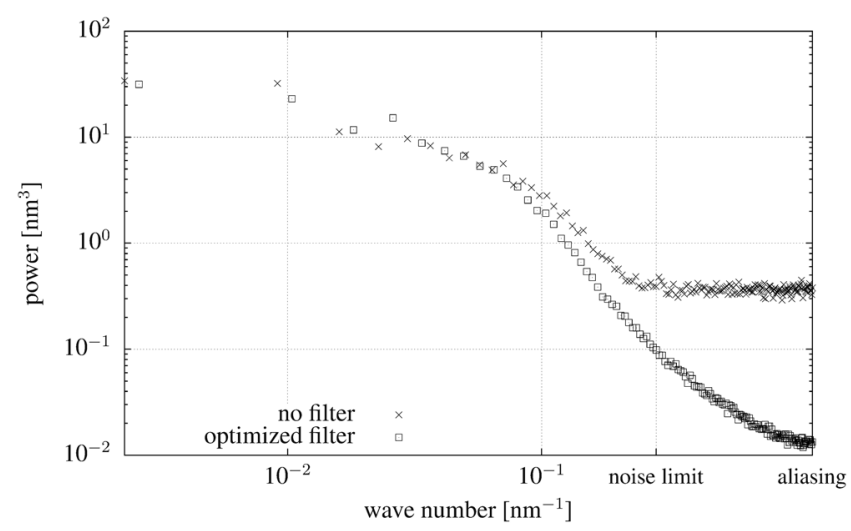

(a)

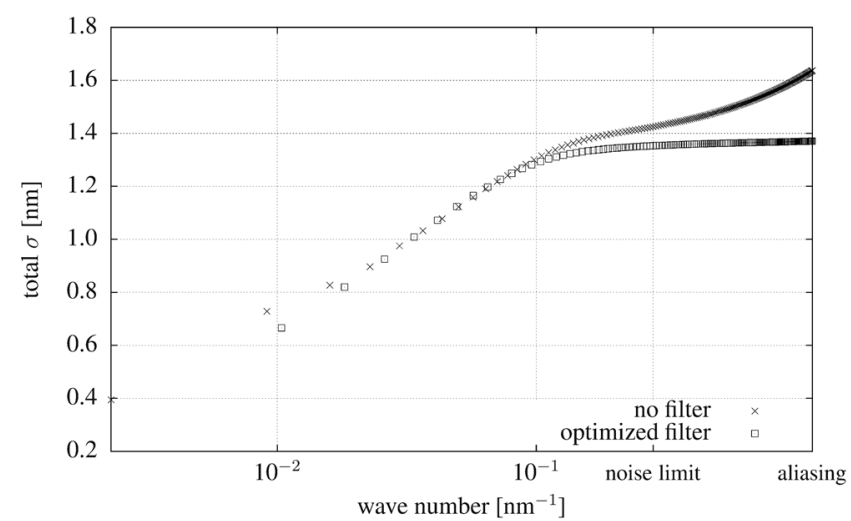

(b)

Fig. 9 A comparison is shown of the PSD and total roughness of filterfree SEM profile-based detection (cross-markers) to optimized elliptic filtered edge detection (square markers). These results are obtained from analyzing the set of SEM images from J. Jussot (see Fig. 1 for an example of one of the images). The PSD is given in (a) and the total roughness is given in (b), which is obtained by taking the square root of the cumulative sum of the PSD. The square markers correspond to the optimized filter found in Figs. 4(a) and 5(a). 
of the approximated SEM signal profile. We expect that the noise level in the PSD [see Fig. 9(a)] could be lowered by correcting the roughness in the SEM signal profile. This ultimately means that we have not yet established the CramérRao lower bound (CRLB). At this point, the LER no longer depends on postprocessing (such as filtering), but has become a function of the pixel noise and, because our estimator is not the CRLB, additional noise due to the specific method of edge determination. This, however, is not a problem because the total noise level can be taken into account ${ }^{8}$ and will be our next point of attention.

\section{Image Noise Analysis}

In estimating the LER [Fig. 9(a)], we used all available images (50 in total) to reduce the uncertainty in the discrete PSD. We now improve this LER determination by considering a model for the PSD and question how much noise is acceptable so that we still can determine LER? In fact, there are two questions: How much noise can we allow in a single image such as Fig. 1 and how many line edges do we need in total? We begin our investigation on the effect of image noise on the determination of LER by generating random rough edges using the model of Palasantzas ${ }^{20}$ with known parameters

$\operatorname{PSD}(k)=\frac{\sqrt{\pi}}{2 \pi} \frac{\Gamma\left(\alpha+\frac{1}{2}\right)}{\Gamma(\alpha)} \frac{2 \sigma^{2} \xi}{\left(1+k^{2} \xi^{2}\right)^{\alpha+\frac{1}{2}}}$.

This PSD defines an infinitely long line with $\sigma$ as the LER, $\xi$ is the correlation length, and $\alpha$ is the roughness exponent. It can be verified that the integral of this PSD equals the variance

$\sigma^{2}=\int_{-\infty}^{+\infty} \operatorname{PSD}(k) \mathrm{d} k$

The random displacements can be generated via the algorithm of Thorsos, which is explained in Ref. 21. The algorithm of Thorsos produces the random edge displacements that, in the limit of large averages, converge toward the PSD of Palasantzas up to a bias in the standard deviation. This bias is explained in Ref. 21 as well and can be compensated for by multiplying the edge displacements with a constant factor.

We generate top-down SEM-like images by using the model of the SEM signal profile obtained earlier (Fig. 7). A SEM-like image is obtained by displacing the SEM signal profile at every row in accordance to the random-generated displacements, which in turn satisfy the PSD given by Eq. (9). If we collect a number of randomly generated lines next to each other in one image, we obtain the result of Fig. 10(a). This is a simulated result of a noise-free top-down SEM-like image of randomly generated line edges. The image is further processed by adding Poisson distributed noise to every pixel of the noise-free image. This Poisson distributed noise is added in a similar way as in Ref. 15. We emphasize that, in contrast to Ref. 15, we did not include Gaussian distributed noise. Examples of Poisson noise-generated SEM-like images are given in Figs. 10(b)-10(d). In each of the images, the average electron density is set to (in respective order) 200, 20, and
2 electrons per pixel. The corresponding average charge density equals (in respective order) 1000,100 , and $10 \mu \mathrm{C}$.

The edges of the random-generated images are determined using filter-free SEM profile-based detection as described before. An example of displacement detection applied to a randomly generated image is given in Fig. 11.

The difference between the randomly generated displacements and the detected displacements after adding pixel noise tells us how pixel noise translates to noise in the edge displacements. This is illustrated in Fig. 12, where the black line corresponds to the randomly generated displacements and the dashed line corresponds to the detected displacements after adding pixel noise. We conclude from running many simulations that this pixel noise translates to a noise distribution in the edge displacements which is uniform (flat) in the PSD. In other words, this means that the pixel noise translates to white noise in the edge displacements.

Now that we have classified the total noise (constituting of pixel noise and additional noise due to our specific method of edge determination), we are free to add this noise term to the Palasantzas model (see also Refs. 8 and 9)

$\operatorname{PSD}_{\mathrm{w} / \text { noise }}(k)=\operatorname{PSD}_{\mathrm{w} / \text { o noise }}(k)+\sigma_{n}^{2} \frac{\Delta y}{2 \pi}$,

where $\sigma_{n}$ is the noise level, and $\Delta y$ is the measurement interval. The PSDs obtained by detecting the edge displacements in simulated SEM-like images with an electron density of 2, 20, and 200 electrons per pixel are given in Fig. 13. In these images, we can also see that the pixel noise translates to white noise: The power of the high frequencies flattens out to a straight line as we decrease the electron density per pixel.

The idea is now to fit the simulated discrete PSD as a function of the number of line edges and to analyze the convergence of the parameters $(\sigma, \xi$, and $\alpha)$ of the Palasantzas model, Eq. (11), extended with a term to capture the total noise $\left(\sigma_{n}\right)$. We remark that by fitting Eq. (11), we neglect the systematic errors described by Ref. 9 . We neglect the systematic errors because in our study, we have a larger number of sampled edge displacements $N$ and the power of the noise level renders the effects due to aliasing or spectral leakage to negligible levels. Our simulation is setup as follows. We generate the random line edges with a length of $2 \mu \mathrm{m}(256 \mathrm{px})$ using the modeled SEM signal profile of Fig. 7. We choose a roughness (LER) of $1.5 \mathrm{~nm}$, correlation length $\xi$ of $25 \mathrm{~nm}$, and a roughness exponent $\alpha$ of 0.75 in the Palasantzas model. These are typical values for experimentally measured edges. ${ }^{8,9}$ Now, we consider the worst case in image noise of the densities given in Fig. 10, which corresponds to an electron density of about 2 electrons per pixel on average (charge density $\approx 10 \mu \mathrm{C}$ ). We run many simulations and determine the distributions of the outcome values of the parameters of the Palasantzas model (LER $\sigma$, noise level $\sigma_{n}$, correlation length $\xi$, and roughness exponent $\alpha$ ). The results of this low-dose (high-noise) simulation are given in Fig. 14.

At first, we observe that all estimated parameters of the Palasantzas model are converging toward the predefined parameters. The convergence, however, seems to be asymptotic, which is best seen by the noise term $\sigma_{n}$ in Fig. 14(b) and the correlation length $\xi$ in Fig. 14(c). There seems to be 


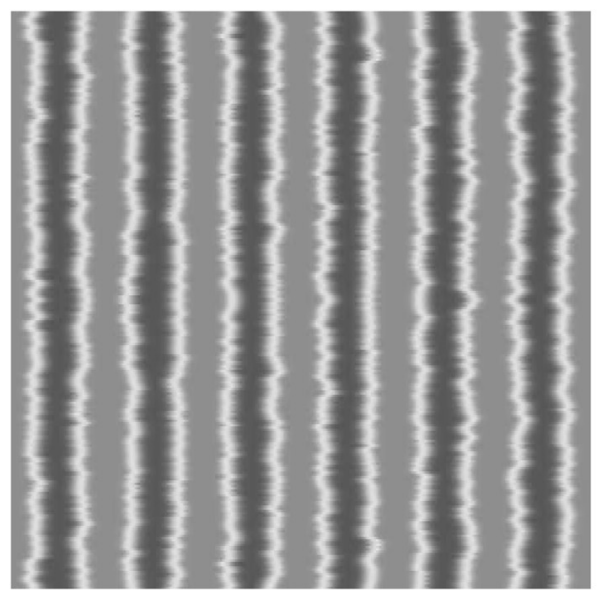

(a) Noise free image.

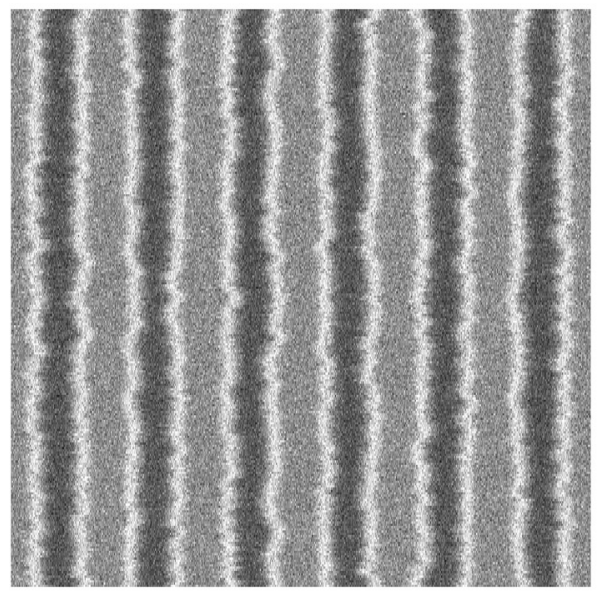

(c) $\langle$ electron density $\rangle \sim 20$ per pixel.

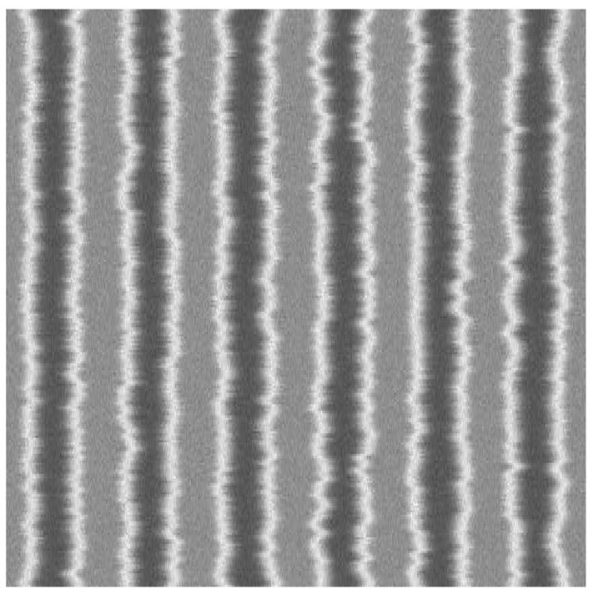

(b) 〈electron density $\sim 200$ per pixel.

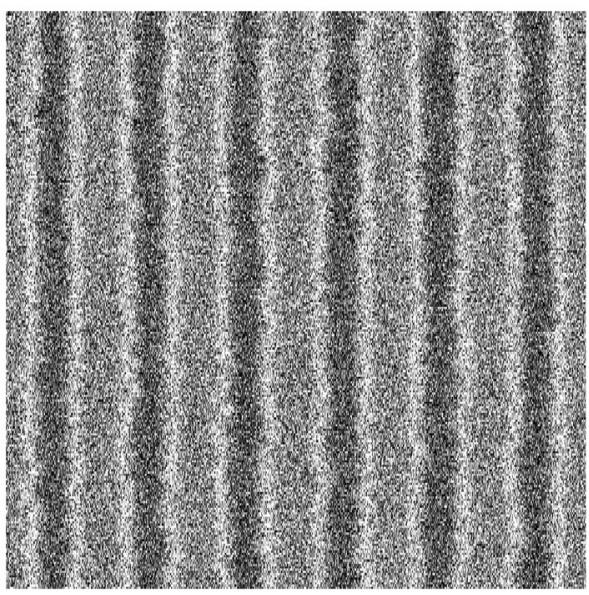

(d) $\langle$ electron density $\rangle \sim 2$ per pixel.

Fig. 10 Randomly generated top-down SEM-like images with line edges. The field size is $2 \mu \mathrm{m}$ in length (256 px) and 450-nm wide (1024 px). The pixel size is about $7 \mathrm{~nm} \times 0.5 \mathrm{~nm}$. (a) Noise-free

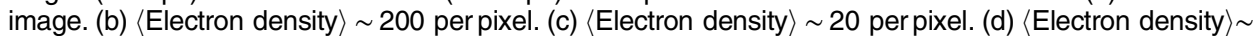
2 perpixel.

a very small bias in the LER, which is best seen in Fig. 14(a). One of the possible causes for this bias is related to the intrinsic bias in the Thorsos algorithm. Although we have numerically corrected this bias in the Thorsos algorithm, it is with limited precision (about three digits). The error bars roughly decrease as the square root of the number of averages, which is to be expected based on averaging principles. The relative errors (size of the error bars divided by the value of the predefined parameter) are given in Fig. 15.

We observe in Fig. 15 that the correlation length $\xi$ and roughness exponent $\alpha$ are harder to estimate, i.e., it takes more averages to produce the same relative error as for LER $\sigma$ and noise level $\sigma_{n}$. The same conclusion can be found in Ref. 15 . The most interesting parameter for industry is LER $\sigma$, because that is the parameter against which process performance is evaluated. When the number of averages is low, the intrinsic noise in the discrete PSD is significant, as can be seen in Fig. 16. It is remarkable that under these conditions, LER can still be estimated with a relative error (onesigma) of about $10 \%$. In other words, it only takes one single image, Fig. 10(d), with 2 electrons per pixel (charge density $\approx 10 \mu \mathrm{C}$ ) to estimate LER as $1.5 \mathrm{~nm} \pm 10 \%$.
We now question how the parameters converge when we increase the electron density per pixel. The effect of this on LER $\sigma$ is given in Fig. 17, where we plot the relative error as a function of the number of line edges for densities of 2, 20, and 200 electrons per pixel.

The result of Fig. 17 shows that it hardly makes any difference if we increase the density from 20 electrons per pixel (charge density $\approx 100 \mu \mathrm{C}$ ) to 200 electrons per pixel (charge density $\approx 1000 \mu \mathrm{C}$ ). The explanation is as follows. We identify that the relative error has two contributions: pixel noise and variance because of limited edge length. This can also be seen by integrating the Palasantzas model given by Eq. (11)

$\sigma_{\mathrm{w} / \text { noise }}^{2}=\sigma_{\mathrm{w} / \mathrm{onoise}}^{2}+\sigma_{n}^{2}$.

The relative error is then determined as

$\operatorname{error}\left(\sigma_{\mathrm{w} / \text { noise }}^{2}\right)=\operatorname{error}\left(\sigma_{\mathrm{w} / \text { onoise }}^{2}\right)+\operatorname{error}\left(\sigma_{n}^{2}\right)$.

In the simulation of an average density of 2 electrons per pixel, we have that both terms in Eq. (13) contribute to the total error. The error due to pixel noise, which is the second 


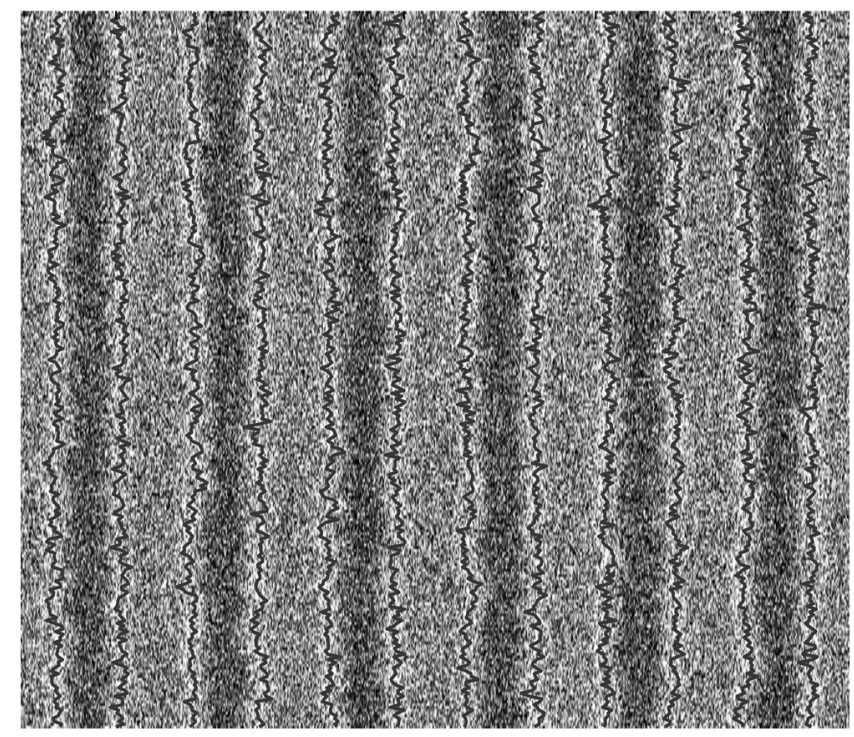

Fig. 11 Demonstration of filter-free SEM profile-based detection applied to a randomly generated top-down SEM-like image with line edges. The field size is $2 \mu \mathrm{m}$ in length (256 px) and 450-nm wide (1024 px). The average electron density of this image is about 2 electrons per pixel and corresponds to an average charge density of about $10 \mu \mathrm{C}$.

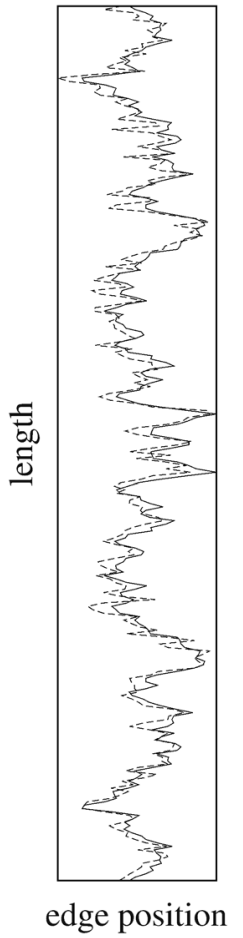

Fig. 12 The randomly generated displacements (solid line) are compared with the detected displacements (dashed line) after adding Poisson distributed pixel noise. The difference between the generated and detected displacements gives the noise distribution in edge detection.

term in Eq. (13), decreases as we increase the electron density per pixel. If we increase the electron density even further, then the variance caused by limited edge length, which is the first term in Eq. (13), becomes significantly larger than the error contribution due to the pixel noise. When we reach this point, the total relative error is primarily determined by the

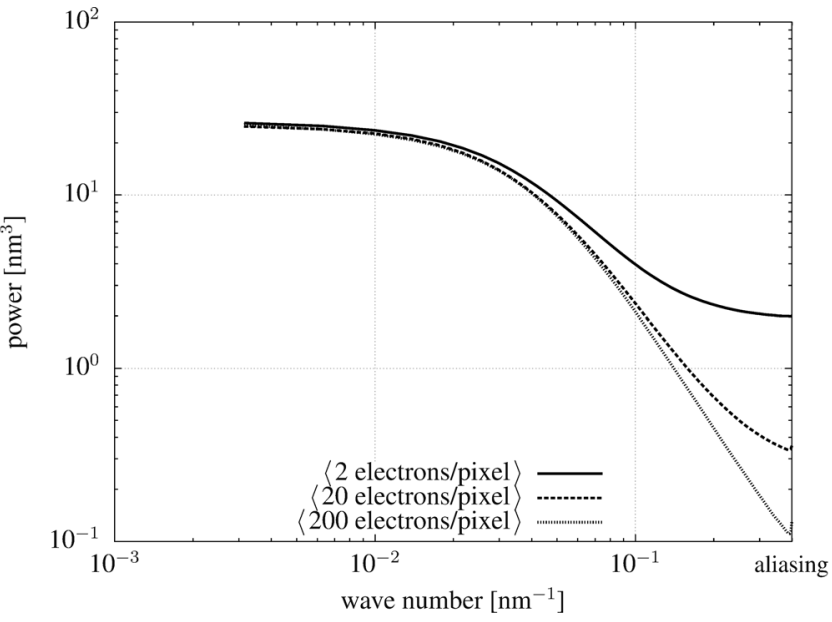

Fig. 13 The effect of adding a noise term to the Palasantzas model is shown. The PSDs in this figure are obtained from averaging many images for different noise settings.

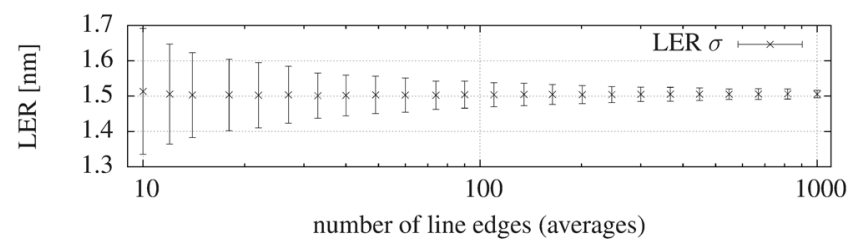

(a)

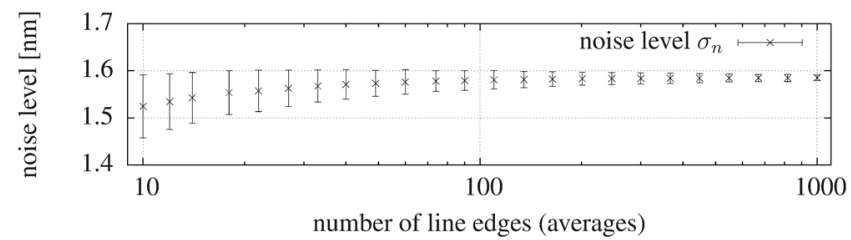

(b)

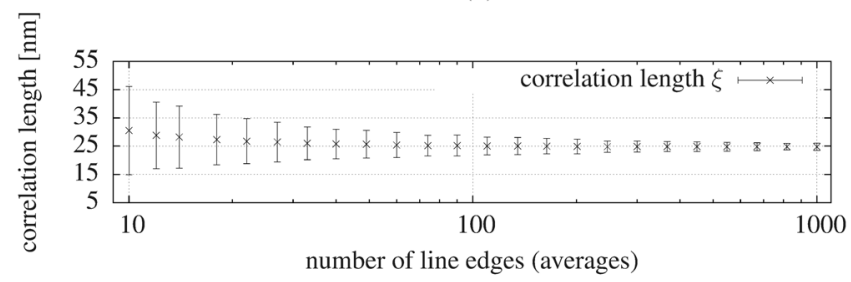

(c)

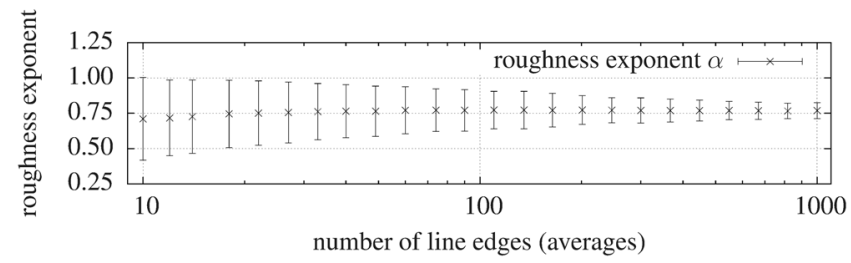

(d)

Fig. 14 The simulation results for fitting the parameters of the Palasantzas PSD model to randomly generated lines after adding Poisson distributed pixel noise are shown. The random lines are generated with a length of $2 \mu \mathrm{m}(256 \mathrm{px})$ using the modeled SEM signal profile of Fig. 7. The generated lines have a roughness (LER) of $1.5 \mathrm{~nm}$, correlation length $\xi$ of $25 \mathrm{~nm}$, and a roughness exponent $\alpha$ equal to 0.75 . The simulated electron density is about 2 electrons per pixel on average. This corresponds to a charge density of approximately $10 \mu \mathrm{C}$. The distribution of outcome values for LER, noise level, correlation length and roughness exponent are given respectively in (a), (b), (c), and (d). 


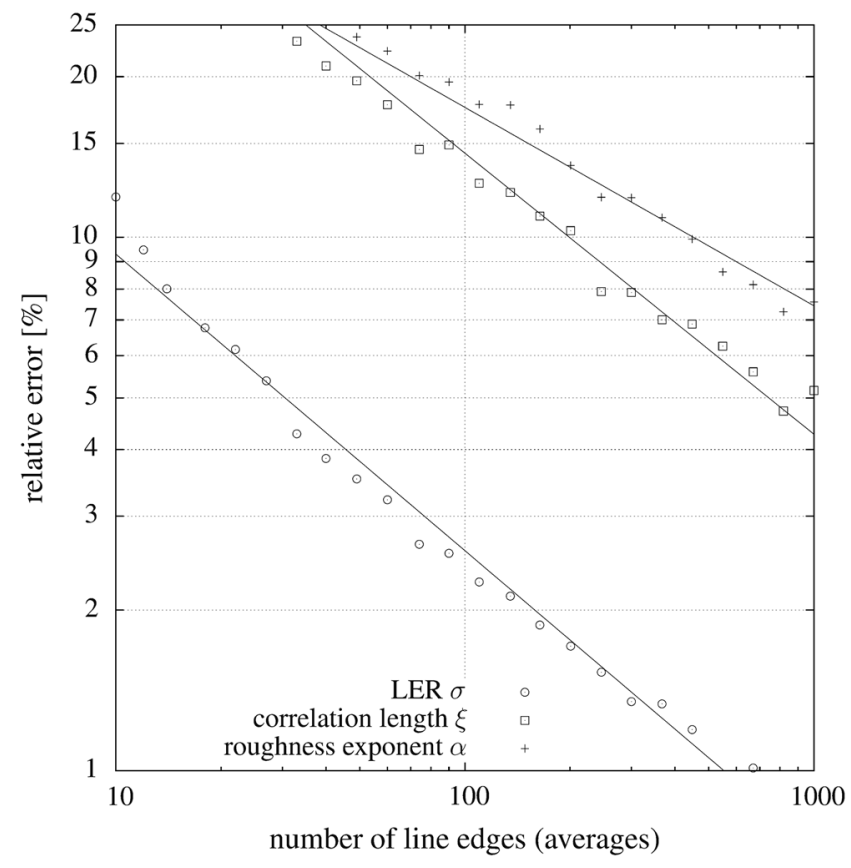

Fig. 15 The relative error (one-sigma) in parameter estimation with respect to the Palasantzas model for simulated top-down SEM-like images with a density of about 2 electrons per pixel on average is shown. The result of this figure is based on the error bars in Fig. 14.

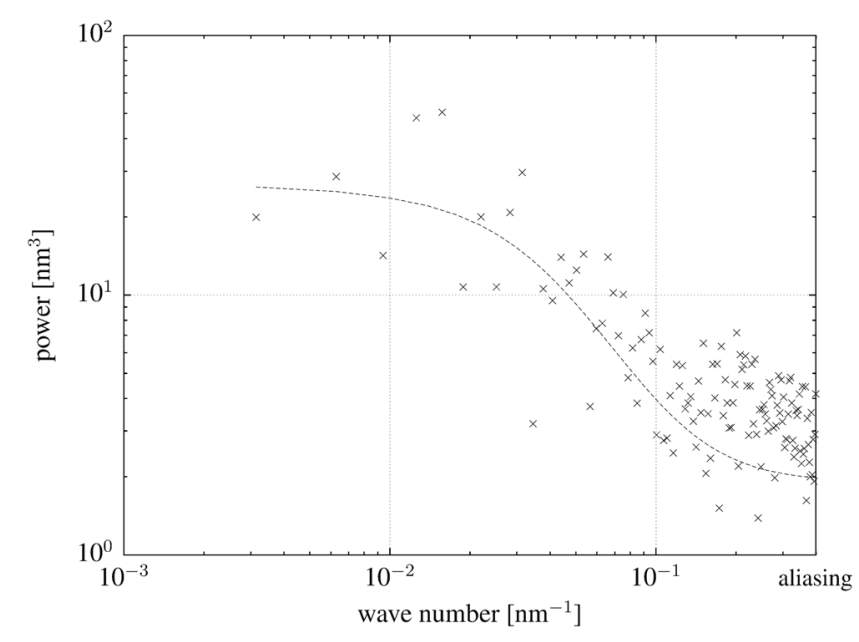

Fig. 16 The intrinsic noise in the discrete PSD after averaging only 10 edges is shown. The dashed line corresponds to the limit of many averages. The data are obtained from a simulation corresponding to a density of about 2 electrons per pixel, which corresponds to a charge density of about $10 \mu \mathrm{C}$.

variance caused by limited edge length. This means that there is no point in further increasing the dose as this does not improve LER determination. In Fig. 17, we see that this already occurs at an averaged density of 20 electrons per pixel.

\section{Conclusion}

The off-line determination of LER in top-down SEM image requires low-noise images. This means that we need a higher dose or we filter the SEM image before edge detection. However, by increasing the dose, we run the risk of resist

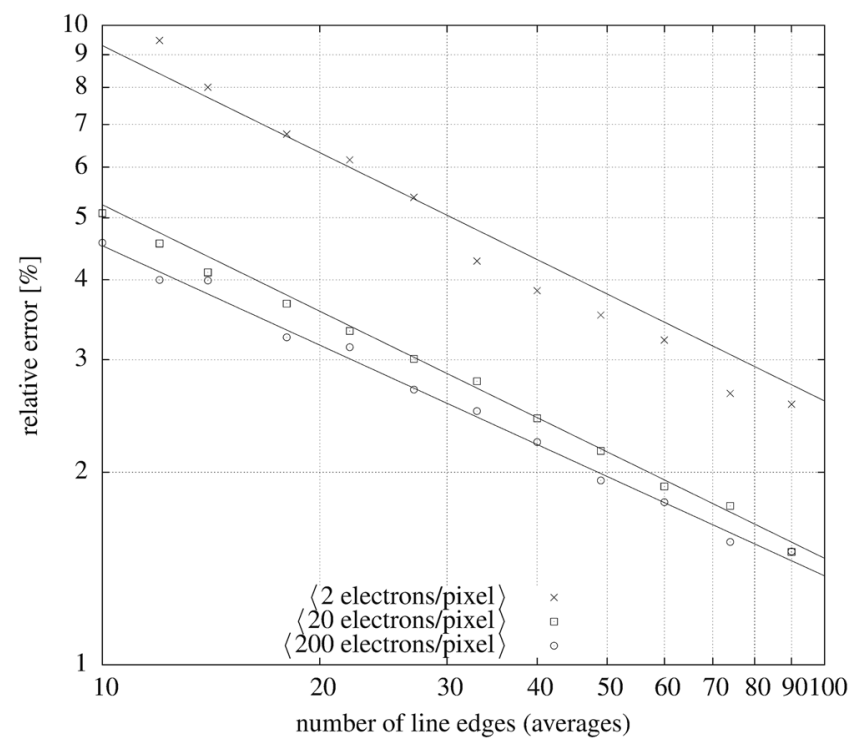

Fig. 17 The relative error in LER determination for simulated topdown SEM-like images with a density of 2, 20, and 200 electrons per pixel on average is shown.

shrinkage. We have shown that the effect of transversal and longitudinal filterings cannot be ignored, even when considering an optimized filter strength. This filter dependency complicates the determination of LER, because the PSD models for LER extraction do not capture this effect. The best solution is to avoid a filter at all.

We developed a different method in which we match the approximated SEM signal profile against the raw (unfiltered) amplitude of edges in a SEM image. The SEM signal profile is obtained by integrating an experimental top-down SEM image of line edges in the direction of the edges. With this method, it is possible to detect the edge displacements in very noisy images without using a filter. In fact, this method works without using any postprocessing of SEM images.

In a simulation study, we show that the LER can still be determined from very noisy images with only 2 electrons per pixel on average (charge density $\approx 10 \mu \mathrm{C}$ ). The PSDs are generally averaged over many line edges to reduce the statistical error. However, even a single image with 12 line edges produces an estimation of the LER with a relative error of only $10 \%$. Furthermore, increasing the dose beyond 20 electrons per pixel does not significantly improve the LER determination. We conclude that with the right analysis, it is possible to get LER with reasonable accuracy at amazingly low dose.

\section{Acknowledgments}

The authors wish to thank J. Jussot from CNRS-LTM/CEALETI for recording the top-down SEM images using a CDSEM. This work is supported by NanoNextNL, a micro- and nanotechnology program of the Dutch Government and 130 partners.

\section{References}

1. A. Yamaguchi, "Characterization of line-edge roughness in resist patterns and estimations of its effect on device performance," Proc. SPIE 5038, 689-698 (2003).

2. J. Lee et al., "Effect of line-edge roughness (LER) and line-width roughness (LWR) on sub-100-nm device performance," Proc. SPIE 5376, 426-433 (2004). 
3. C. Gustin et al., "Impact of line width roughness on the matching performances of next-generation devices," Thin Solid Films 516(11), 36903696 (2008).

4. G. P. Patsis et al., "Quantification of line-edge roughness of photoresists. I. A comparison between off-line and on-line analysis of topdown scanning electron microscopy images," J. Vac. Sci. Technol. B: Microelectron. Nanometer Struct. 21(3), 1008-1018 (2003).

5. T. Ohfuji, M. Endo, and H. Morimoto, "Theoretical analysis of line-edge roughness using FFT techniques," Proc. SPIE 3678, 732-738 (1999).

6. A. Hiraiwa and A. Nishida, "Discrete power spectrum of line width roughness," J. Appl. Phys. 106(7), 074905 (2009).

7. A. Nishida, "Statistical- and image-noise effects on experimental spectrum of line-edge and line-width roughness," J. Micro/Nanolithogr., MEMS, MOEMS 9(4), 041210 (2010).

8. L. Azarnouche et al., "Unbiased line width roughness measurements with critical dimension scanning electron microscopy and critical dimension atomic force microscopy," J. Appl. Phys. 111(8), 084318 (2012).

9. C. A. Mack, "Systematic errors in the measurement of power spectral density," J. Micro/Nanolithogr., MEMS, MOEMS 12(3), 033016 (2013).

10. A. Yamaguchi et al., "Bias-free measurement of LER/LWR with low damage by CD-SEM" Proc. SPIE 6152, 61522D (2006).

11. A. Yamaguchi et al., "Single-shot method for bias-free LER/LWR evaluation with little damage," Microelectron. Eng. 84(5-8), 17791782 (2007)

12. T. Ohashi et al., "Precise measurement of photoresist cross-sectional shape change caused by SEM-induced shrinkage," Proc. SPIE $\mathbf{8 6 8 1}$, $86810 \mathrm{~K}$ (2013).

13. A. Yamaguchi and J. Yamamoto, "Influence of image processing on line-edge roughness in CD-SEM measurement," Proc. SPIE 6922 692221 (2008).

14. A. Hiraiwa, "Image-pixel averaging for accurate analysis of line-edge and linewidth roughness," J. Micro/Nanolithogr., MEMS, MOEMS 10(2), 023010 (2011)

15. V. Constantoudis and E. Pargon, "Evaluation of methods for noise-free measurement of LER/LWR using synthesized CD-SEM images," Proc. SPIE 8681, 86812L (2013).

16. J. S. Villarrubia and B. D. Bunday, "Unbiased estimation of linewidth roughness," Proc. SPIE 5752, 480-488 (2005).

17. A. Hiraiwa and A. Nishida, "Statistically accurate analysis of line width roughness based on discrete power spectrum," Proc. SPIE 7638, $76380 \mathrm{~N}(2010)$.
18. P. Kruit and S. Steenbrink, "Shot noise in electron-beam lithography and line-width measurements," Scanning 28(1), 20-26 (2006).

19. T. F. Coleman and Y. Li, "An interior trust region approach for nonlinear minimization subject to bounds," SIAM J. Optim. 6(2), 418-445 (1996).

20. G. Palasantzas, "Roughness spectrum and surface width of self-affine fractal surfaces via the K-correlation model," Phys. Rev. B 48(19), 472 478 (1993).

21. C. A. Mack, "Generating random rough edges, surfaces, and volumes," Appl. Opt. 52(7), 1472-1480 (2013).

Thomas Verduin is a PhD student at Delft University of Technology and specializes in the field of computational physics. In 2009, he received his bachelor of applied physics at Delft University of Technology, The Netherlands. He then went to Utrecht University to complete a master's study in theoretical physics (2013). In 2012, he started a PhD degree to investigate the shotnoise in e-beam lithography in the Charged Particle Optics Group of Delft University of Technology.

Pieter Kruit is a full professor of physics at Delft University of Technology in The Netherlands. He is (co-) author of over 150 publications in refereed international journals, author of 50 international patents, and supervisor of $30 \mathrm{PhD}$ dissertations. He has had research programs on nanometer-resolution electron spectroscopy, low energyspread electron and ion sources, and multibeam optics for microscopy and lithography. He is cofounder of MAPPER Lithography, a company developing maskless electron beam lithography, and DELMIC.

Cornelis W. Hagen received a master's degree (1983) and a PhD degree (1991) from the Free University of Amsterdam, The Netherlands. He was a researcher at the Paul Scherrer Institute in Switzerland from 1989 to 1992, and at the Kamerlingh Onnes Laboratory of Leiden University, The Netherlands, from 1992 to 1994. In 1994, he joined Delft University of Technology as an assistant professor and was appointed as associate professor in 2008. His area of research is microscopy and lithography with charged particles. 